7-1-1985

\title{
Incident Beam Polarization for Laser Doppler Velocimetry Employing a Sapphire Cylindrical Window
}

James A. Lock

Cleveland State University, j.lock@csuohio.edu

Harold J. Schock

Follow this and additional works at: https://engagedscholarship.csuohio.edu/sciphysics_facpub

Part of the Physics Commons

How does access to this work benefit you? Let us know!

\section{Publisher's Statement}

This paper was published in Applied Optics and is made available as an electronic reprint with the permission of OSA. The paper can be found at the following URL on the OSA website: http://www.opticsinfobase.org/ao/abstract.cfm?URI=ao-24-13-1987. Systematic or multiple reproduction or distribution to multiple locations via electronic or other means is prohibited and is subject to penalties under law.

\section{Original Citation}

Lock, James A. and Harold J. Schock. "Incident Beam Polarization for Laser Doppler Velocimetry Employing a Sapphire Cylindrical Window." Applied Optics 24 (1985): 1987-1995.

\section{Repository Citation}

Lock, James A. and Schock, Harold J., "Incident Beam Polarization for Laser Doppler Velocimetry Employing a Sapphire Cylindrical Window" (1985). Physics Faculty Publications. 47.

https://engagedscholarship.csuohio.edu/sciphysics_facpub/47

This Article is brought to you for free and open access by the Physics Department at EngagedScholarship@CSU. It has been accepted for inclusion in Physics Faculty Publications by an authorized administrator of EngagedScholarship@CSU. For more information, please contact library.es@csuohio.edu. 


\title{
Incident beam polarization for laser Doppler velocimetry employing a sapphire cylindrical window
}

\author{
James A. Lock and Harold J. Schock
}

\begin{abstract}
For laser Doppler velocimetry studies employing sapphire windows as optical access ports, the birefringency of sapphire produces an extra beam intersection volume which serves to effectively smear the acquired velocity flow field data. It is shown that for a cylindrical window geometry, the extra beam intersection volume may be eliminated with minimal decrease in the fringe visibility of the remaining intersection volume by suitably orienting the polarizations of the initial laser beams. For horizontally incident beams, these polarizations were measured at three intersection locations within the cylinder. It was found that the measured polarization angles agreed with the theoretical predictions.
\end{abstract}

\section{Introduction}

In the past, LDV measurements on motored pistons employing a full $360^{\circ}$ optical access cylindrical chamber have been confined to nonfiring configurations using thin Plexiglas ${ }^{1,2}$ or fused silicon ${ }^{3}$ cylinders. For these situations, the optics of the laser beams passing through the cylinder is relatively straightforward. However, such materials are prone to scratching due to the motion of the piston ring against the cylinder wall. For firing configurations, thin Plexiglas and quartz cylinders do not suffice since they cannot withstand pressures and temperatures associated with combustion. These mechanical problems are largely overcome by employing a sapphire cylinder.4,5 It has been determined that, although a thin sapphire cylinder may not withstand the pressures associated with combustion, ${ }^{5}$ a relatively thick sapphire cylinder would be able to withstand the thermal stresses produced if the cylinder is only intermittently fired.

However, in spite of its potential mechanical convenience, sapphire is an optically complicated material since it possesses a small degree of birefringence. If a laser beam is passed through the wall of a sapphire cylinder, at the surface of the sapphire the refracted ray breaks into two components, the ordinary and extraordinary rays. These two rays propagate in different directions within and beyond the cylinder wall. Applying this to LDV studies, if two converging laser

James A. Lock is with Cleveland State University, Physics Department, Cleveland, Ohio 44115; Harold J. Schock is with NASA Lewis Research Center, Cleveland, Ohio 44135.

Received 11 February 1985. beams pass through the sapphire cylinder before their intersection point, the birefringence produces two distinct intersection volumes possessing interference fringes. One is due to the intersection of the two ordinary rays, and the other is due to the intersection of the two extraordinary rays. These intersection volumes are separated by up to $114-\mu \mathrm{m} / \mathrm{in}$. thickness of the sapphire cylinder. Since the diameters of the intersection volumes are typically of the order of $25 \mu \mathrm{m}$, a smeared sampling of the flow pattern results. If both fringe volumes are used for flow measurement, any measured flow velocity is a weighted average of the flow velocities in the two measurement volumes. On the other hand, for a thick cylinder with the fringe volumes far enough apart, it is likely that the LDV photodetector system is tuned finely enough so that it might only register one of the two fringe volumes. Since each intersection volume contains only a fraction of the incident laser power, the counting rate resulting from registering only one of the intersection volumes could be very low. If the birefringence of the sapphire cylinder could be effectively negated, and a single fringe volume produced, obtaining maximally efficient counting rates would be guaranteed. Examination of the birefringency of sapphire shows that such effective negating is possible.

Sapphire possesses a single optic axis, and the ordinary and extraordinary rays within the sapphire are each linearly polarized. The polarization rotations experienced by the ordinary and extraordinary rays within the material are determined by the direction of the propagation of the incident light, the direction of the optic axis, and the direction of the normal to the window surfaces and are independent of the polarization of the incident light. Thus it is possible to orient the polarization of an incident laser beam so that within the sapphire its polarization direction is identical to the 
polarization direction of either the ordinary or extraordinary ray. For such a matching of initial conditions, the light transmitted into the sapphire will have $100 \%$ of its amplitude fed into one of the two modes and $0 \%$ of its amplitude fed into the other of the two modes. Although both modes of propagation within the sapphire are mathematically and physically allowed, the initial conditions are arranged so that only one of them occurs.

This arrangement of initial polarizations produces a single fringe volume. Due to the requirement of matching the initial conditions at the outer surface of the window, the two converging electric fields will not be parallel at the intersection volume. This produces a contrast loss in the interference pattern at the intersection volume again resulting in decreased counting rates. It is the purpose of this paper to demonstrate that there exist particular optical alignments which cause this contrast loss to be virtually zero at the same time that the problem of cylinder birefringence is circumvented. This means that in many situations it is more advantageous to go through the effort of suitably orienting the input optics and the cylinder to eliminate one of the fringe volumes than it is to ignore the birefringence and measure either the smeared flow field or the flow field in only one of the fringe volumes. If the birefringence is ignored, the counting rates will certainly be lower, but if the birefringence is compensated for, the decrease in counting rate can be made negligible. As a result, we claim that sapphire is a viable material from which to machine optical access windows for hightemperature LDV studies. Its good mechanical properties may be enjoyed at the same time that its optical complications may be largely overcome. To make it convenient for other investigators to adapt our formulation to their own cylinder geometries, a relatively complete presentation of the calculation details is included in this paper.

\section{Cylindrical Window Geometry}

We consider a single crystal of sapphire which has been machined to form a hollow right circular cylinder of outer radius $r$ and wall thickness $a$. For purposes of analysis, the cylinder is oriented so that its axis of symmetry is along the $z$ axis of a reference coordinate system, and its optic axis $\hat{N}$ makes an angle $\theta$ with the $z$ axis, and its projection onto the $x, y$ plane makes an angle $\phi$ with the $x$ axis. The laser beams are incident on the cylinder with their bisector parallel to the $x$ axis and with the beams making angles of convergence $\eta$ with the center line. This geometry is shown for horizontally incident beams in Fig. 1(a) and for vertically incident beams in Fig. 1(b).

A light ray passing through such a cylinder has its initial refraction $i$ at the exterior surface of the cylinder and its final refraction $f$ at the interior surface of the cylinder. At the initial refraction, the inward normal to the cylinder surface is $\hat{n}_{i}$, the incident light propagation direction is $\hat{k}_{i}$ making an angle $\gamma_{i}$ with $\hat{n}_{i}$, and the transmitted light propagation direction is $\hat{k}_{o}$ making an angle $\gamma_{o}$ with $\hat{n}_{i}$. At the final refraction, the inward normal to the cylinder surface is $\hat{n}_{f}$, the incident light propagation direction is $\hat{k}_{o}$ making an angle $\gamma_{h}$ with $\hat{n}_{f}$, and the transmitted light propagation direction is $k_{f}$ making an angle of $\gamma_{f}$ with $\hat{n}_{f}$. If $n$ is the index of refraction for sapphire appropriate to the particular mode to be transmitted, Snell's law gives

$$
\begin{aligned}
\sin \gamma_{i} & =n \sin \gamma_{o}, \\
n \sin \gamma_{h} & =\sin \gamma_{f}
\end{aligned}
$$

for either the ordinary or approximately for the extraordinary mode since the birefringence of sapphire is low. Similarly, the ordinary ray and extraordinary ray polarizations within the sapphire are given by

$$
\begin{aligned}
& \hat{D}_{\text {ord }}=\left(\hat{k}_{o} \times \hat{N}\right) / \sin \xi, \\
& \hat{D}_{\text {ext }} \approx \hat{k}_{o} \times \hat{D}_{\text {ord }},
\end{aligned}
$$

where $\xi$ is the angle between $\hat{k}_{o}$ and $\hat{N} .{ }^{6}$

At an interface, the polarization direction of nonnormally incident light suffers a rotation on either refraction or reflection. Such rotations are calculated by

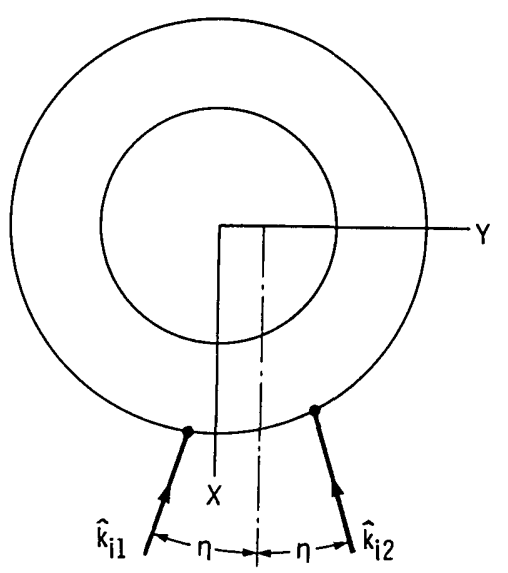

(a) Hor izontally converging beams.

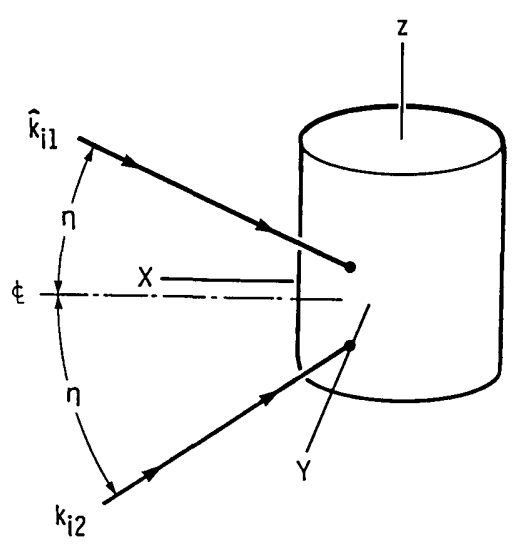

(b) Vertically converging beams.

Fig. 1. (a) Horizontally converging laser beams incident on a sapphire cylinder. The convergence angle of the beams is $\eta$. (b) Vertically converging laser beams incident on a sapphire cylinder. The convergence angle of the beams is $\eta$. 


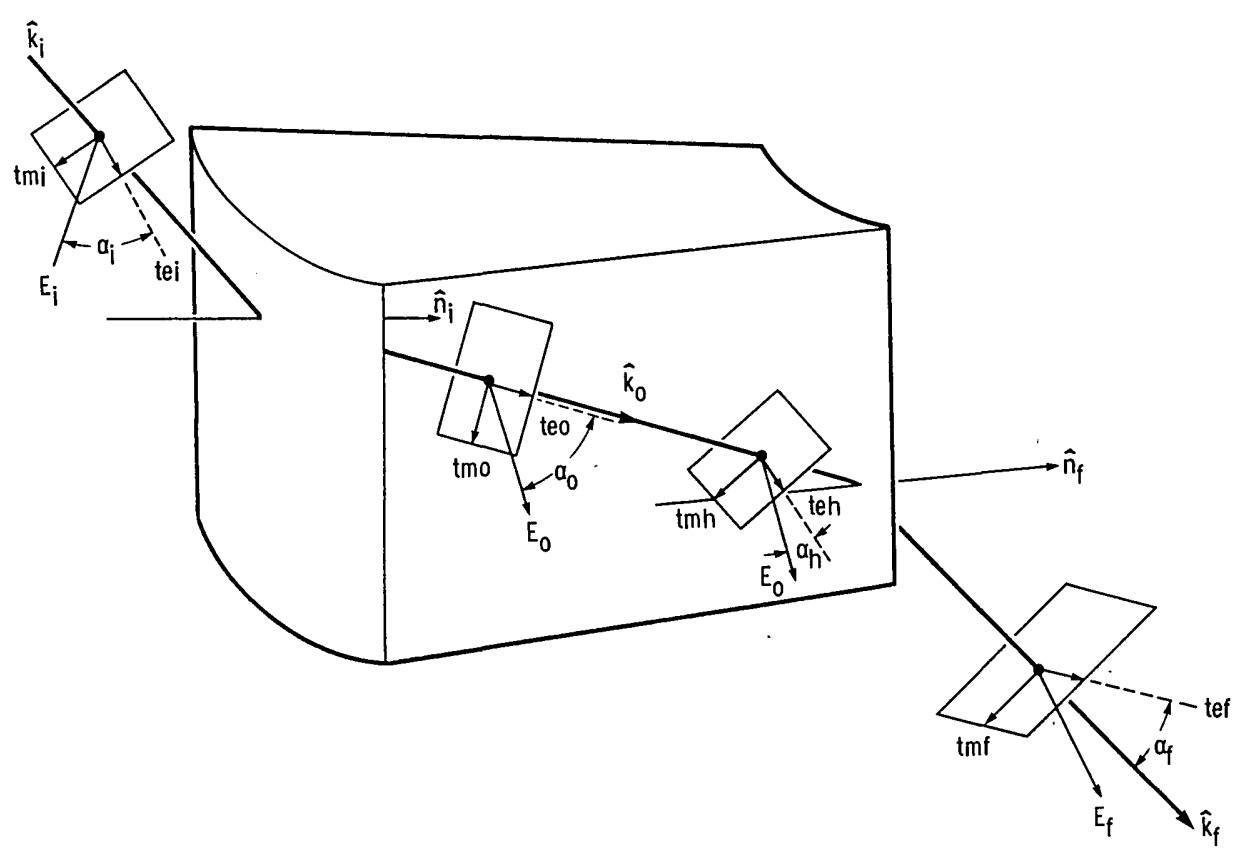

Fig. 2. Transverse electric te and transverse magnetic tm components of a laser beam at the outside and inside interfaces of a sapphire cylinder. decomposing the incident light into its transverse electric te (also known as the $s$ polarization) and transverse magnetic $t m$ (also known as the $p$ polarization) components, determining the amount of refraction or reflection of each of these components and then recombining the new amplitudes after the interface. The te mode is parallel to the plane of the interface and perpendicular to the direction of propagation of the light ray (i.e., $\hat{k} \times \hat{n}$ ), and the $t m$ mode is perpendicular to both the direction of propagation of the light ray and the $\hat{t} e$ direction (i.e., $\hat{k} \times \hat{t} e$ ). For our case, the te and $\mathrm{tm}$ directions before the initial interface are denoted by tê $i$ and $t \hat{m} i$, and the angle that the initial field $\mathbf{E}_{i}$ makes with the tê $i$ axis is $\alpha_{i}$. This geometry is indicated in Fig. 2. After the first interface, the $t e$ and $t m$ directions are denoted by têo and $t \hat{m} o$, and the angle that the transmitted field $\mathbf{E}_{o}$ makes with têo is $\alpha_{o}$. Before the second interface, the $t e$ and $t m$ directions are denoted by têh and $t \hat{m} h$, and the angle that $\mathbf{E}_{o}$ makes with têh is $\alpha_{h}$. After the second interface, the te and $t m$ directions are denoted by $t \hat{e} f$ and $t \hat{m} f$, and the angle that the final field $\mathbf{E}_{f}$ makes with $t \hat{e} f$ is $\alpha_{f}$.

The components of an incident electric field normalized to unit magnitude may be written as

$$
\mathbf{E}_{i}=\cos \alpha_{i} t \hat{e} i+\sin \alpha_{i} t \hat{m} i \text {. }
$$

Then the components of the electric field transmitted at the first interface are

$$
\mathbf{E}_{o}=F_{e} \cos \alpha_{i} t \hat{e} o+F_{m} \sin \alpha_{i} t \hat{m} o,
$$

where ${ }^{7}$

$$
\begin{aligned}
F_{e} & =\frac{2 \cos \gamma_{i}}{\cos \gamma_{i}+n \cos \gamma_{o}}=\frac{2 \cos \gamma_{i} \sin \gamma_{o}}{\sin \left(\gamma_{i}+\gamma_{o}\right)}, \\
F_{m} & =\frac{2 \cos \gamma_{i}}{n \cos \gamma_{i}+\cos \gamma_{o}}=\frac{2 \cos \gamma_{i} \sin \gamma_{o}}{\sin \left(\gamma_{i}+\gamma_{o}\right) \cos \left(\gamma_{i}-\gamma_{o}\right)} .
\end{aligned}
$$

Equation (6) may be rewritten as

$$
\mathbf{E}_{o}=\frac{F_{e} \cos \alpha_{i}}{\cos \alpha_{o}}\left(\cos \alpha_{o} t \hat{e} o+\sin \alpha_{o} t \hat{m} o\right),
$$

where the polarization angle of the transmitted field $\alpha_{o}$ is given by

$$
\tan \alpha_{o}=\frac{\tan \alpha_{i}}{F_{e} / F_{m}} .
$$

Our goal is to have $\mathbf{E}_{o}$ be along the direction of $\hat{D}_{\text {ord }}$ or $\hat{D}_{\text {ext }}$ to insure that $100 \%$ of the transmitted amplitude is in the desired mode and that $0 \%$ of the transmitted amplitude is in the other mode. To place $\mathbf{E}_{o}$ along the $\hat{D}_{\text {ord }}$ direction requires that

$$
\begin{aligned}
\tan \alpha_{o, \text { ord }} & =\frac{t \hat{m} o \cdot \hat{D}_{\text {ord }}}{t \hat{e} o \cdot \hat{D}_{\text {ord }}}=-\frac{\hat{N} \cdot t \hat{e} i}{\hat{N} \cdot\left(\hat{k}_{o} \times t \hat{e} i\right)} \\
& =\frac{\hat{k}_{i} \cdot\left(\hat{N} \times \hat{n}_{i}\right)}{\left(\hat{N} \cdot \hat{k}_{i}\right) \cos \gamma_{o}-\left(\hat{N} \cdot \hat{n}_{i}\right) \cos \left(\gamma_{i}-\gamma_{o}\right)} .
\end{aligned}
$$

This result is pleasing in that the appropriate incident polarization direction for the laser beam may be calculated from Eqs. (10) and (11) without having to know explicitly anything about the direction of the beam inside the sapphire. By similar analysis, if one wished the polarization of the refracted ray to be coincident with $\hat{D}_{\text {ext }}$, the initial polarization would be aligned to the angle $\alpha_{i}$ given by Eq. (10) with

$$
\alpha_{o, \text { ext }}=\alpha_{o, \text { ord }}+\pi / 2 .
$$

For treatment of the final interface, the electric field within the sapphire may be rewritten as

$$
\mathbf{E}_{o}=\frac{F_{e} \cos \alpha_{i}}{\cos \alpha_{o}}\left(\cos \alpha_{h} t \hat{h} h+\sin \alpha_{h} t \hat{m} h\right) .
$$

In analogy with the treatment of the initial interface, after transmission through the final interface the final electric field becomes 


$$
E_{f}=\frac{F_{e} \cos \alpha_{i}}{\cos \alpha_{o}} \frac{F_{e}^{\prime} \cos \alpha_{h}}{\cos \alpha_{f}}\left(\cos \alpha_{f} t \hat{e} f+\sin \alpha_{f} t \hat{m} f\right),
$$

where

$$
\begin{aligned}
F_{e}^{\prime} & =\frac{2 \sin \gamma_{f} \cos \gamma_{h}}{\sin \left(\gamma_{f}+\gamma_{h}\right)}, \\
F_{m}^{\prime} & =\frac{2 \sin \gamma_{f} \cos \gamma_{h}}{\sin \left(\gamma_{f}+\gamma_{h}\right) \cos \left(\gamma_{f}-\gamma_{h}\right)}, \\
\tan \alpha_{f} & =\frac{\tan \alpha_{h}}{F_{e}^{\prime} / F_{m}^{\prime}}, \\
\tan \alpha_{h, \text { ord }} & =\frac{t \hat{m} h \cdot \hat{D}_{\text {ord }}}{t \hat{e} h \cdot \hat{D}_{\text {ord }}}=-\frac{\hat{N} \cdot t \hat{e} f}{\hat{N} \cdot\left(\hat{k}_{o} \times t \hat{e} f\right)} \\
& =\frac{\hat{k}_{f} \cdot\left(\hat{N} \times \hat{n}_{f}\right)}{\left(\hat{N} \cdot \hat{k}_{f}\right) \cos \gamma_{h}-\left(\hat{N} \cdot \hat{n}_{f}\right) \cos \left(\gamma_{f}-\gamma_{h}\right)}, \\
\alpha_{h, \text { ext }} & =\alpha_{h, \text { ord }}+\pi / 2 .
\end{aligned}
$$

For calculating the power level in the intersection volume of the two laser beams, it is notationally convenient to rewrite the final electric field of Eq. (14) as

$$
\mathbf{E}_{f}=E_{f} \hat{u}_{f},
$$

where the amplitude of the final field is

$$
E_{f}=F_{e} F_{e}^{\prime} \frac{\cos \alpha_{i} \cos \alpha_{h}}{\cos \alpha_{o} \cos \alpha_{f}}
$$

and where

$$
\hat{u}_{f}=\cos \alpha_{f} t \hat{e} f+\sin \alpha_{f} t \hat{m} f
$$

is the unit vector in the direction of the final electric field. At a point within the intersection volume of beams 1 and 2 , the total electric field is then

$$
\mathbf{E}_{\text {total }}=E_{f 1} \hat{u}_{f 1}+\exp (i \Phi) E_{f 2} \hat{a}_{f 2},
$$

where the $\exp (i \Phi)$ factor describes the positionally dependent relative phases of the two intersecting electric fields.

Then the total intensity is

$$
I_{\text {total }}=E_{\text {total }}^{*} \cdot \mathbf{E}_{\text {total }}=E_{f 1}^{2}+E_{f 2}^{2}+2 \cos \Phi E_{f 1} E_{f 2}\left(\hat{u}_{f 1} \cdot \hat{u}_{f 2}\right) .
$$

The fringe visibility is defined by

$$
V=\frac{I_{\max }-I_{\min }}{I_{\max }+I_{\min }}
$$

where $I_{\max }$ and $I_{\min }$ are the maximum and minimum intensities obtained by varying the relative phase factor $\Phi$ between constructive and destructive interference. In the intersection volume of the two beams, we obtain

$$
V=\left(\frac{2 E_{f 1} E_{f 2}}{E_{f 1}^{2}+E_{f 2}^{2}}\right)\left(\hat{u}_{f 1} \cdot \hat{u}_{f 2}\right),
$$

where

$$
\begin{aligned}
\hat{u}_{f 1} \cdot \hat{u}_{f 2}= & \cos \alpha_{f 1} \cos \alpha_{f 2} t \hat{e} f_{1} \cdot t \hat{e} f_{2} \\
& +\cos \alpha_{f 1} \sin \alpha_{f 2} t \hat{e} f_{1} \cdot t \hat{m} f_{2} \\
& +\sin \alpha_{f 1} \cos \alpha_{f 2} t \hat{m} f_{1} \cdot t \hat{e} f_{2} \\
& +\sin \alpha_{f 1} \sin \alpha_{f 2} t \hat{m} f_{1} \cdot t \hat{m} f_{2} .
\end{aligned}
$$

The first factor of Eq. (26) describes losses in contrast due to the inequality of the individual field amplitudes $E_{f 1}$ and $E_{f 2}$. Such losses in contrast also occur in nonbirefringent windows since the two beams travel different paths and undergo different losses on their way to the intersection volume. The second factor of Eq. (26) describes losses in contrast due to $\mathbf{E}_{f 1}$ and $\mathbf{E}_{f 2}$ not being parallel. This is the factor of interest in the analysis of birefringent windows. Since we chose to align $\mathbf{E}_{o 1}$ and $\mathbf{E}_{o 2}$ along the directions of $\hat{D}_{\text {ord, } 1}$ and $\hat{D}_{\text {ord, } 2}$ or along $\hat{D}_{\text {ext, } 1}$ and $\hat{D}_{\text {ext, } 2}$ to eliminate one of the propagation modes and its corresponding second LDV measurement volume, $\mathbf{E}_{f 1}$ and $\mathbf{E}_{f 2}$ will in general not be parallel. This results in $\left|\hat{u}_{f 1} \cdot \hat{u}_{f 2}\right|<1$ and losses in contrast beyond those described by the first factor of Eq. (26).

\section{Ray Tracing for Horizontally and Vertically Incident Beams}

In the previous section it was seen that both the initial beam polarizations and the fringe contrast in the intersection volume depend on the geometry of the laser beams outside the cylinder and within the cylinder cavity. However, for each location of the beam intersection within the cylinder this geometry changes. Thus it is necessary to perform a ray tracing calculation for the laser beams to determine this dependence of the beam geometry on the location of the beam intersection.

In this section such a ray tracing calculation is carried out for a particular example of a cylinder-optical system configuration. We assume that the cylinder is fixed in space in an upright position and that the entire laser velocimeter system plus final lens is mounted on a movable table. The table is free to move in the horizontal plane. In this example we assume that after leaving a laser velocimeter optical system, the horizontally incident or vertically incident laser beams are parallel, and each is a distance $\Delta$ from the common center line. These beams are then incident on the final converging lens of focal length $F$. After passing through this lens, the angle of convergence of the beams is $\eta$ where

$$
\Delta / F=\tan \eta,
$$

and their resulting intersection point is taken to be located at the origin of the fixed coordinate system described in Sec. II and shown in Fig. 1. The cylinder is then placed so that its symmetry axis is coincident with the $z$ axis. After insertion of the cylinder, the horizontally incident beams continue to intersect at the origin, while vertically incident beams now have their intersection point shifted to a location on the negative $x$ axis. This is a result of the cylinder acting as an astigmatic lens for the incident beams. ${ }^{8}$

As mentioned before, in this specific example the laser velocimeter plus final lens system is mounted on a movable table. Translations of the table in the $Y$ direction with respect to the table's initial position will be denoted by $\tau$. Similarly, translations of the table in the $X$ direction with respect to the table's initial position will be denoted by $\epsilon$. By suitably moving the table 
while keeping the cylinder fixed and thereby varying $\tau$ and $\epsilon$, the intersection point of either the horizontal beams or vertical beams may be moved to any fixed coordinates $(x, y)$ within the cylinder.

The first beam geometry considered is that of two converging laser beams in the horizontal plane with their center line parallel to the $x$ axis as is shown in Fig. 1(a). Beam 1 is incident from the left, and beam 2 is incident from the right as seen from above. Let $\beta_{j}$ be the angle between the $x$ axis and a cylinder radius which passes through the outer diameter of the cylinder as shown in Fig. 3. Then

$$
\gamma_{i j}=\beta_{j} \pm \eta,
$$

where the upper sign is associated with $j=1$, the left incident ray, and the lower sign is associated with $j=$ 2 , the right incident ray. The index $i$ means incident at the initial interface as shown in Fig. 2.

The equation of the outer surface of the cylinder is

$$
x^{2}+y^{2}=r^{2},
$$

and the equation of beam 1 outside the cylinder is

$$
y=-(x-\epsilon) \tan \eta+\tau .
$$

These two curves intersect when

$$
\sin \gamma_{i 1}=\frac{\tau}{r} \cos \eta+\frac{\epsilon}{r} \sin \eta
$$

A similar calculation for beam 2 gives

$$
\sin \gamma_{i 2}=\frac{\tau}{r} \cos \eta-\frac{\epsilon}{r} \sin \eta
$$

Inside the cylinder cavity the equation for each beam is

$$
y=x \tan \psi_{j}+\frac{r \sin \gamma_{i j}}{\cos \psi_{j}}
$$

where

$$
\begin{aligned}
\sin \gamma_{h j} & =\left(\frac{r}{r-a}\right) \frac{\sin \gamma_{i j}}{n}, \\
\psi_{j} & =\gamma_{i j}-\gamma_{o j}+\gamma_{h j}-\gamma_{f j} \mp \eta .
\end{aligned}
$$

This geometry is shown in Fig. 3. The two interior beams intersect when

$$
\begin{aligned}
& \frac{x}{r}=\frac{\sin \gamma_{i 1} \cos \psi_{2}-\sin \gamma_{i 2} \cos \psi_{1}}{\sin \left(\psi_{2}-\psi_{1}\right)}, \\
& \frac{y}{r}=\frac{\sin \gamma_{i 1} \sin \psi_{2}-\sin \gamma_{i 2} \sin \psi_{1}}{\sin \left(\psi_{2}-\psi_{1}\right)} .
\end{aligned}
$$

Equations (37) and (38) may be inverted numerically to give $\gamma_{i 1}$ and $\gamma_{i 2}$ and via Eqs. (32) and (33) to give $(\tau, \epsilon)$ as a function of $(x, y)$.

The second beam geometry considered is that of two converging laser beams in a plane parallel to the $x-z$ plane as shown in Fig. 1(b). Beam 1 is incident from above, and beam 2 is incident from below. If viewed from above as in Fig. 4 , the angle $\beta_{i}$ is the angle that the intersection of the laser beam and the outer surface of the cylinder makes with respect to the $x$ axis. A geometric difficulty is encountered in this case which was not present in the case of the two horizontal beams.
Although $\hat{k}_{i}$ is in a plane parallel to the $x-z$ plane, the refracted ray $\hat{k}_{o}$ within the sapphire is not in this same plane but acquires a small $Y$ component due to the refraction. The plane of incidence for the initial refraction is not the same as the plane of incidence for the final refraction.

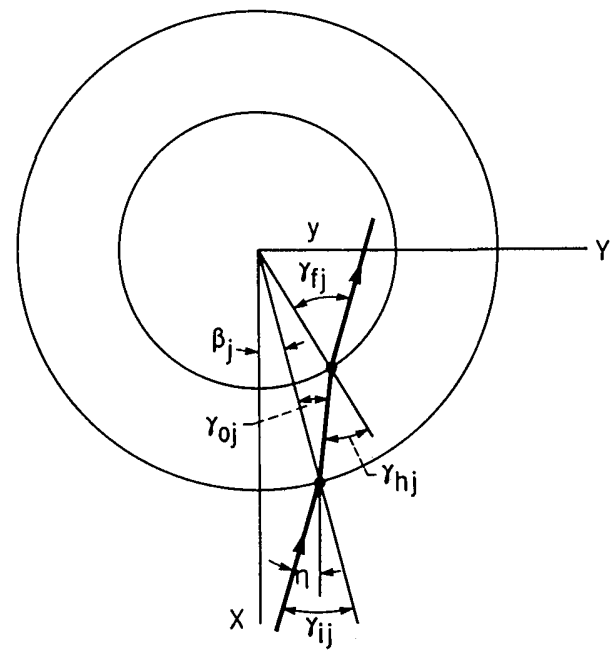

Fig. 3. Angles of incidence and refraction for a horizontally incident laser beam passing through a sapphire cylinder.

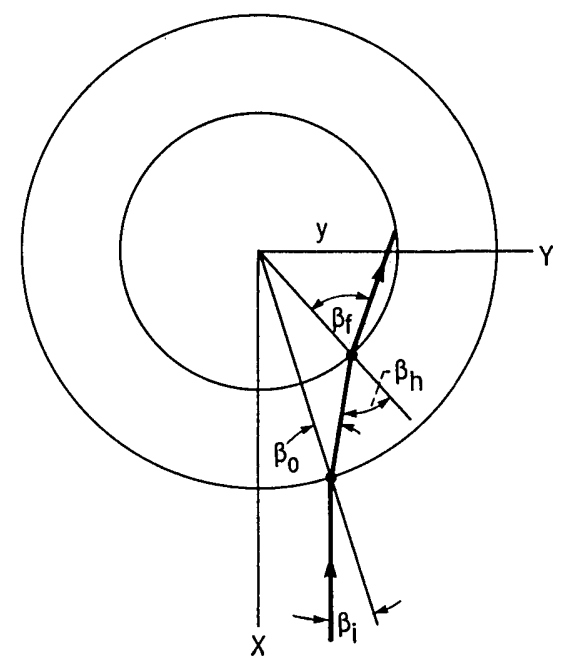

(a) Projection from above.

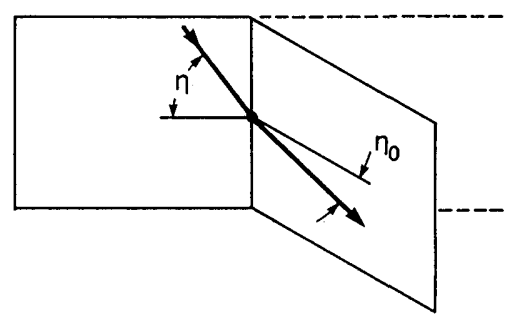

(b) Projection from the side.

Fig. 4. (a) View from above for a vertically incident laser beam passing through a sapphire cylinder. (b) View from the side for a vertically incident laser beam passing through a sapphire cylinder. 
Due to this complication, the simplest way to view the refraction of the vertically incident beams is to consider the geometry in two orthogonal views. First, we consider $\hat{k}_{i}$ and $\hat{k}_{o}$ as seen from above in Fig. 4(a). Second, we consider $\hat{k}_{i}$ and $\hat{k}_{o}$ as seen from the directions perpendicular to the vertical planes which contain $\hat{k}_{i}$ and $\hat{k}_{o}$, respectively, as shown in Fig. $4(\mathrm{~b})$. The calculation of $\hat{k}_{o}$ for Fig. 4 gives

$$
\begin{aligned}
& \cos \gamma_{i}=\cos \beta_{i} \cos \eta, \\
& \sin \beta_{o}=\frac{\sin \beta_{i} \cos \eta}{\left(n^{2}-\sin ^{2} \eta\right)^{1 / 2}}, \\
& \sin \beta_{h}=\left(\frac{r}{r-a}\right) \sin \beta_{o}, \\
& \sin \eta_{o}=(\sin \eta) / n,
\end{aligned}
$$

where again $\gamma_{i}$ is the angle of incidence at the initial interface from Eq. (1). The equation of beam 1 outside the cylinder is

$$
\left\{\begin{array}{l}
z=(x-\epsilon) \tan \eta \\
y=\tau .
\end{array}\right.
$$

Its intersection with the outer surface of the cylinder occurs at

$$
\begin{aligned}
y & =\tau, \\
z_{i} & =\dot{\tan } \eta\left[\left(r^{2}-\tau^{2}\right)^{1 / 2}-\epsilon\right] .
\end{aligned}
$$

Within the cylinder the beam vertically drops a distance

$$
z_{o}=\tan \eta_{o}\left[r \cos \beta_{o}-(r-a) \cos \beta_{h}\right],
$$

and the intersection of the beam with the interior surface of the cylinder occurs at

$$
\begin{aligned}
& x_{f}=(r-a) \cos \mu, \\
& y_{f}=(r-a) \sin \mu,
\end{aligned}
$$

where

$$
\mu=\beta_{i}-\beta_{o}+\beta_{h} .
$$

Once inside the cylinder cavity, the projection of the beam travels a horizontal distance:

$$
L=\left(z_{i}-z_{o}\right) / \tan \eta
$$

before crossing the $x-y$ plane. Thus the intersection point of the two vertically incident beams is

$$
\begin{aligned}
& \frac{x}{r}=\frac{x_{f}}{r}-\frac{L}{r} \cos \psi, \\
& \frac{y}{r}=\frac{y_{f}}{r}-\frac{L}{r} \sin \psi,
\end{aligned}
$$

where for this vertically incident geometry, the angle $\psi$ is

$$
\psi=\beta_{i}-\beta_{o}+\beta_{h}-\beta_{f}
$$

and similarly

$$
\sin \beta_{f}=\left(\frac{r}{r-a}\right) \sin \beta_{i} .
$$

Again Eqs. (51) and (52) may be inverted numerically to give $(\tau, \epsilon)$ as a function of $(x, y)$.

\section{Polarizations for the Horizontally and Vertically Incident Beams}

To have the horizontally incident beams of Fig. 1(a) propagate solely in the extraordinary mode, we require from Eqs. (11) and (12) that

$$
\alpha_{o j}=\arctan \left[\frac{1}{\tan \theta \sin \left(\beta_{j}-\phi-\gamma_{o j}\right)}\right]+\pi / 2
$$

For $j=1,2$, with $\alpha_{i j}$ then being determined from $\alpha_{o j}$ by Eq. (10). In Eq. (55), $\theta$ is the angle that the optic axis makes with the $z$ axis, and $\phi$ is the angle that the projection of the optic axis onto the $x-y$ plane makes with the $x$ axis. The geometry of the horizontal beam configuration is especially simple since the beams remain in the horizontal plane for their entire traverse. As a result

$$
\text { têi } i=t \hat{e} o=t e \hat{h}=t \hat{e} f=\hat{u}_{z},
$$

where $\hat{u}_{z}$ is the unit vector along the $z$ axis,

$$
\begin{aligned}
t \hat{m} o & =t \hat{m} h, \\
\alpha_{h j} & =\arctan \left[\frac{1}{\tan \theta \sin \left(\epsilon_{j}-\phi-\gamma_{h j}\right)}\right]+\pi / 2, \\
\epsilon_{j} & =\gamma_{i j}-\gamma_{o j}+\gamma_{h j} \mp \eta, \\
\hat{u}_{f 1} \cdot \hat{u}_{f 2} & =\cos \alpha_{f 1} \cos \alpha_{f 2}+\sin \alpha_{f 1} \sin \alpha_{f 2} \cos \left(\psi_{1}-\psi_{2}\right) .
\end{aligned}
$$

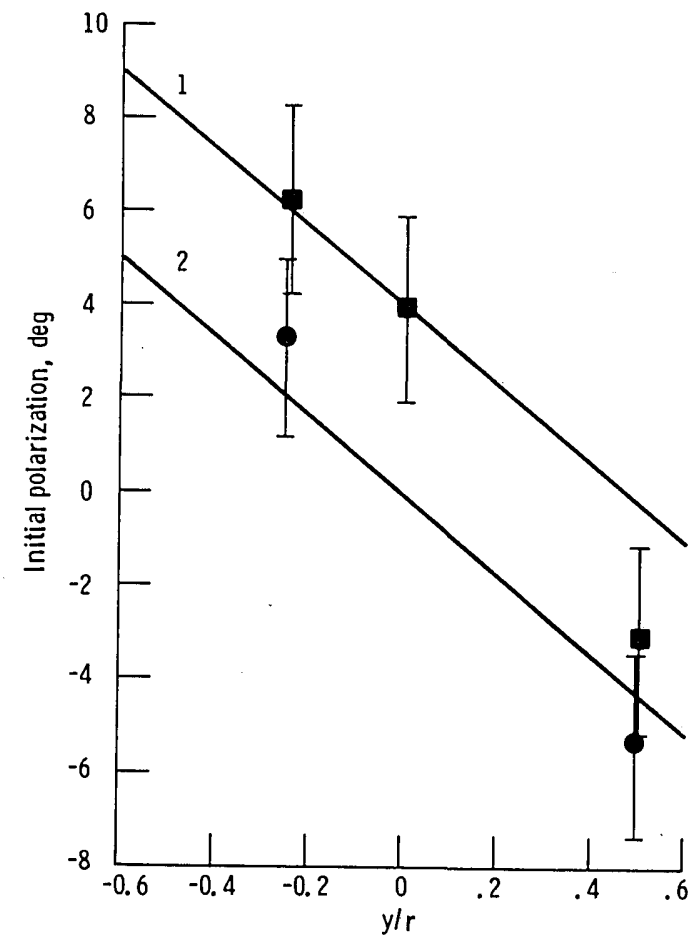

Fig. 5. Polarization angles required to propagate the horizontally incident beams 1 and 2 solely in the extraordinary mode as a function of $y / r$ for $x / r=0, \theta=18^{\circ}$, and $\phi=0^{\circ}$. The polarization angles are referenced with respect to the polarization of beam 2 at $x=y=0$. The reference angle itself is at an angle of $2.07^{\circ}$ counterclockwise from the vertical direction. Positive angles represent clockwise rotations as seen from along the direction of $\hat{k}$. The five data points are the measurements of Sec. V. 
To have the vertically incident beams of Fig. 1(b) propagate solely in the ordinary mode, we require from Eqs. (11) and (12) that

$$
\tan \alpha_{o j}=\frac{\cos \theta \cos \eta \sin \beta_{i} \mp \sin \theta \sin \eta \sin \left(\beta_{i}-\phi\right)}{(\cos \eta \sin \theta \cos \phi \pm \sin \eta \cos \theta) \cos \gamma_{o j}-\sin \theta \cos \left(\beta_{i}-\phi\right) \cos \left(\gamma_{i j}-\gamma_{o j}\right)} .
$$

Due to $\hat{k}_{o j}$ acquiring a small $Y$ component within the sapphire, no simplifications for $\alpha_{h j}$ and the te and $t m$ dot products analogous to Eqs. (56)-(60) are possible for the vertically incident beams. As a result we obtain

$$
\tan \alpha_{h j}=\frac{\cos \theta \cos \eta \sin \beta_{f} \mp \sin \theta \sin \eta \sin (\mu-\phi)}{[\cos \eta \sin \theta \cos (\psi-\phi) \pm \sin \eta \cos \theta] \cos \gamma_{h j}-\sin \theta \cos (\mu-\phi) \cos \left(\gamma_{o h}-\gamma_{o f}\right)},
$$

$$
t \hat{e} f_{1} \cdot t \hat{e} f_{2}=1-\frac{2 \sin ^{2} \eta}{\cos ^{2} \eta \sin ^{2} \beta_{f}+\sin ^{2} \eta},
$$

$t \hat{e} f_{1} \cdot t \hat{m} f_{2}=-t \hat{m} f_{1} \cdot t \hat{e} f_{2}=\frac{2 \sin \eta \cos ^{2} \eta \sin \beta_{f} \cos \beta_{f}}{\cos ^{2} \eta \sin ^{2} \beta_{f}+\sin ^{2} \eta}$,

$t \hat{m} f_{1} \cdot t \hat{m} f_{2}=1-\frac{2 \sin ^{2} \eta \cos ^{2} \eta \cos ^{2} \beta_{f}}{\cos ^{2} \eta \sin ^{2} \beta_{f}+\sin ^{2} \eta}$.

\section{Polarization Measurements}

The Intermittent Combustion Engine Branch of NASA Lewis Research Center had purchased a cylindrically machined single crystal of sapphire from Crystal Systems, Inc., with the parameters

$$
\begin{aligned}
& r=6.033 \mathrm{~cm} \text { (2.375 in.), } \\
& a=1.588 \mathrm{~cm} \text { (0.625 in.). }
\end{aligned}
$$

The optic axis of this cylinder has been previously measured to be at

$$
\theta=18^{\circ}
$$

with respect to the symmetry axis of the sapphire. ${ }^{9}$ The cylinder was illuminated with horizontally converging laser beams at the $0.5145-\mu \mathrm{m}$ wavelength of an argonion laser. For this wavelength, the ordinary and extraordinary indices of refraction of sapphire were taken to be ${ }^{10,11}$

$$
\begin{aligned}
& n_{o}=1.774, \\
& n_{e}=1.766 .
\end{aligned}
$$

The laser beams were measured to have a convergence angle relative to the beam bisector of

$$
\eta=6.37^{\circ} .
$$

To test the polarization predictions of Secs. II-IV, the sapphire cylinder was rotated to the $\phi=0^{\circ}$ orientation. This was done both because $\phi=0^{\circ}$ is interferometrically simple to locate ${ }^{9}$ and because $\alpha_{i 1}$ and $\alpha_{i 2}$ take on their maximum ranges at this angle. It was thought that it would be easier to measure the polarization angles if they varied significantly throughout the cylinder than if they were relatively insensitive to the beam intersection location as is the case for $\phi=90^{\circ}$, for example. With these input data, the polarization angles $\alpha_{i 1}$ and $\alpha_{i 2}$ for propagating the horizontally incident beams solely in the extraordinary mode were calculated as a function of $y / r$ for $x / r=0$ and appear in Fig. 5. The fringe visibility $V$ for the extraordinary mode propa-

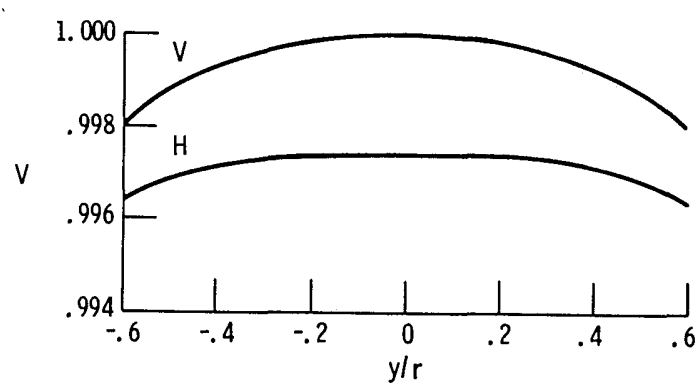

Fig. 6. Fringe visibility obtained from propagating the horizontally incident beams $H$ solely in the extraordinary mode and vertically incident beams $V$ solely in the ordinary mode as a function of $y / r$ for $x / r=0, \theta=18^{\circ}$, and $\phi=0^{\circ}$.

gation of the horizontally incident beams was also calculated as a function of $y / r$ for $x / r=0$ and appears in Fig. 6. The $y$ axis was chosen for calculation since LDV measurements along this line within a cylinder using horizontally and vertically incident laser beams correspond to measurement of the radial and longitudinal components of an axisymmetric flow field, respectively. (For an axisymmetric flow, the angular component of the flow is constant by symmetry.)

The horizontally incident beams were made to intersect at the three locations within the cylinder,

$$
\left(\frac{x}{r}, \frac{y}{r}\right)=(0.00,-0.25),(0.00,0.00),(0.00,0.50),
$$

and the initial polarization direction required to propagate each beam solely in the extraordinary mode was measured at each location. To eliminate the uncertainty involved in obtaining exactly vertical polarization as a reference angle for the above measurements, all the polarization angles were measured with respect to the angle which produced solely extraordinary propagation of beam 2 for the center location. These measured polarizations are also shown in Fig. 5. 
There are two major sources of uncertainty in the experimental measurements. The intensity of the ordinary focal waist as a function of initial polarization angle $\alpha_{i}$ is

$$
I_{\text {ord }}\left(\alpha_{i}\right)=I_{\max } \sin ^{2}\left(\alpha_{i}-\alpha_{i, \text { ext }}\right) .
$$

The minimum of this function is fairly broad, and as a result there is $\sim 1^{\circ}$ uncertainty in determining the exact position of the relative minimum. Second, for every $1^{\circ}$ of rotation of the polarization rotator dial, the actual polarization of the laser light was changed by $2^{\circ}$. Since the polarization rotator dial could not be read any more accurately than to $1^{\circ}$, an additional $\pm 1^{\circ}$ uncertainty was produced in the measured polarization angle. Taking these uncertainties into account, it is seen in Fig. 5 that four of the five data points agree with the theoretical predictions to within the experimental uncertainties.

With the intent of performing future simultaneous two-component LDV measurements employing such a sapphire cylinder, the initial polarizations required to propagate vertically incident laser beams solely in the ordinary mode and the associated fringe visibility were calculated for the parameters of Eqs. (66)-(69),(71) and as a function of $y / r$ for $x / r=0$ and appear in Figs. 7 and 6 , respectively. Again it is calculated that the fringe visibility should be well over $99 \%$. It should finally be noted that if the other alternative was attempted, i.e., causing the horizontally incident beams to propagate solely in the ordinary mode and the vertically incident beams to propagate solely in the extraordinary mode, the resulting fringe visibility would be lower by $\sim 94 \%$.

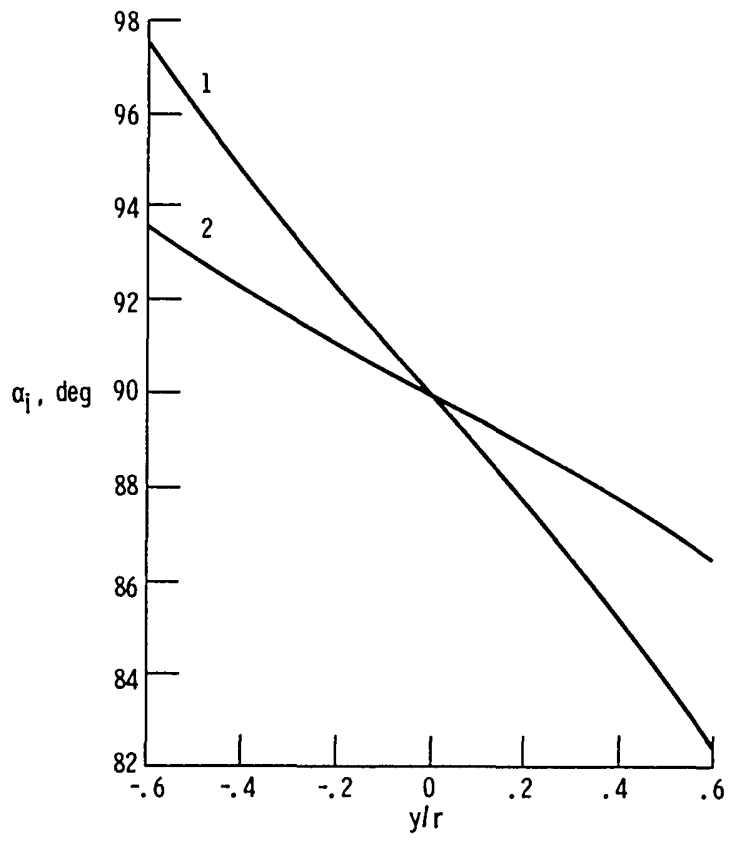

Fig. 7. Polarization angles required to propagate the vertically incident beams 1 and 2 solely in the ordinary mode as a function of $y / r$ for $x / r=0, \theta=18^{\circ}$, and $\phi=0^{\circ}$. The polarization angles are referenced with respect to the projection of the vertical direction on the plane perpendicular to $\hat{k}$. Positive angles represent clockwise rotations as seen from along the direction of $\hat{k}$.

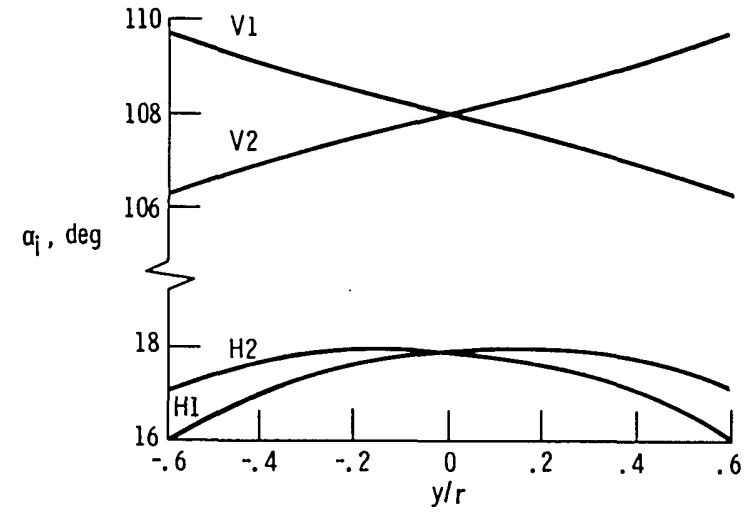

Fig. 8. Polarization angles required to propagate the horizontally incident beams $H 1$ and $H 2$ solely in the extraordinary mode and the vertically incident beams $V 1$ and $V 2$ solely in the ordinary mode as a function of $y / r$ for $x / r=0, \theta=18^{\circ}$, and $\phi=90^{\circ}$.

\section{Discussion}

In Sec. I it was mentioned that for firing configurations for motored pistons, the mechanical and thermal difficulties encountered could be overcome by employing a sapphire cylinder. However, the sapphire introduced optical complications, which as we have seen in this paper may also be overcome. The $\phi=0^{\circ}$ orientation of the cylinder was examined in Sec. V for two reasons. First, this orientation is interferometrically simple to obtain. Second, it predicts a large variation of initial polarizations, and this large variation is easy to observe and permits a relatively straightforward experimental testing of the polarization formulas. For actual LDV applications, it may well be more convenient to employ the $\phi=90^{\circ}$ configuration. This orientation is also interferometrically simple to obtain, and, as seen in Fig. 8, the polarization angles $\alpha_{i 1}$ and $\alpha_{i 2}$ vary minimally as the location of the beam intersection is changed. Thus for $\phi=90^{\circ}$ the polarization angles may be set at some average value at the outset, and measurements with these angles may be made with high-fringe visibility at any location within the cylinder.

Last, if $\theta$ is considered as a free parameter, calculations based on Eqs. (55)-(65) show that the largest fringe visibilities over the greatest ranges of $x / r$ and $y / r$ occur when $\theta=0^{\circ}$. Thus it is most advantageous for dual-beam laser velocimetry applications to machine the sapphire cylinder so that its optic axis is as close as possible to being along its axis of symmetry.

\section{References}

1. C. Arcoumanis, A. F. Bicen, and J. H. Whitelaw, "Effect of Inlet Parameters on the Flow Characteristics in a Four-Stroke Model Engine," SAE Technical Paper 820750 (June 1982).

2. C. Arcoumanis, A. F. Bicen, and J. H. Whitelaw, "Measurements of a Motored Four-Stroke Reciprocating Model Engine," J. Fluid Eng. 104, 235 (1982).

3. H. J. Schock, C. A. Regan, W. J. Rice, and R. A. Chlebecek, "Multicomponent Velocity Measurement in a Piston-Cylinder Configuration Using Laser Velocimetry," TSI Quarterly 11, Issue 4, 3 (1983). 
4. P. O. Witze and R. R. Vilchis, "Stroboscopic Laser Shadowgraph Study of the Effect of Swirl on Homogeneous combustion in a Spark Ignition Engine," SAE Technical Paper 810226 (23-27 Feb. 1981).

5. R. M. Richman and W. C. Reynolds, "The Flow Diagnostics Engine: A New System for Piston Engine Research," Stanford U. Technical Report FDE-1 (Dec. 1982).

6. M. Born and E. Wolf, Principles of Optics (Pergamon, New York, 1980), Sec. 14.3.

7. J. R. Reitz, F. J. Milford, and R. W. Christy, Foundations of Electromagnetic Theory (Addison-Wesley, Reading, Mass., 1979), Sec. 18.2

8. H. J. Schock, S. K. Case, and L. Konicek, "Window Aberration Correction in Laser Velocimetry Using Multifaceted Holographic Optical Elements," Appl. Opt. 23, 752 (1984).

9. J. A. Lock, H. J. Schock, and C. A. Regan, "The Determination of the Direction of the Optic Axis of Sapphire Windows," NASA Technical Paper TM 86892 (to be published).

10. I. H. Malitson, "Refraction and Dispersion of Synthetic Sapphire," J. Opt. Soc. Am. 52, 1377 (1962).

11. M. A. Jeppesen, "Some Optical, Thermo-Optical, and PiezoOptical Properties of Synthetic Sapphire," J. Opt. Soc. Am. 48, 629 (1958).

Meetings Calendar continued from page 1956

1986

February

10-15 4th Int. Symp. on Quantum Optics, Hamilton D. Walls, U. of Waikato, Physics Dept., Hamilton, New Zealand

13-14

Optical Fiber Sensors, OSA Top. Mtg., San Diego OSA Mtgs. Dept., 1816 Jefferson Pl., N.W., Wash., D.C. 20036

24-26

Optical Fiber Communication Conf., Atlanta OSA Mtgs. Dept., 1816 Jefferson Pl., N.W., Wash., D.C. 20036

26-28 Integrated \& Guided-Wave Optics Top. Mtg., Atlanta OSA Mtgs. Dept., 1816 Jefferson Pl., N.W. Wash., D.C. 20036

March

2-7 Astronomical Instrumentation Confs., Tucson SPIE P.O. Box 10, Bellingham, Wash. 98227

9-14 Microlithography Conf., Santa Clara SPIE, P.O. Box 10, Bellingham, Wash. 98227

10-14 The Pittsburgh Conf., Atlantic City W. Howe, 12 Federal Dr., Suite 322, Pittsburgh, Pa. 15235

24-27 Short Wavelength Coherent Radiation OSA Top. Mtg., Monterey OSA Mtgs. Dept., 1816 Jefferson Pl. N.W., Wash., D.C. 20036

31-2 Apr. Quantum-Limited Imaging \& Image Processing, Top. Mtg., Honolulu OSA Mtgs. Dept., 1816 Jefferson Pl., N.W., Wash., D.C. 20036

31-2 Apr. Holography Top Mtg., Honolulu OSA Mtgs. Dept., 1816 Jefferson Pl., N.W., Wash., D.C. 20036

31-4 Apr. Optics \& Electro-Optics, 1986 Tech. Symp. Southeast, Orlando SPIE, P.O. Box 10, Bellingham, Wash. 98227
31-4 Apr. Optics \& Electro-Optics, 1986 Tech. Symp. East, Arlington SPIE, P.O. Box 10, Bellingham, Wash. 98227

31-5 Apr. Spring '86, Honolulu OSA Mtgs. Dept., 1816 Jefferson Pl., N.W., Wash., D.C. 20036

April

2-5 Signal Recovery \& Synthesis II Top. Mtg., Honolulu OSA Mtgs. Dept., 1816 Jefferson Pl., N.W., Wash., D.C. 20036

3-4 Meteorological Optics Top. Mtg., Honolulu OSA Mtgs. Dept., 1816 Jefferson Pl., N.W., Wash., D.C. 20036

13-23 3rd Int. Conf. on Optical \& Electro-Optical Applied Science \& Engineering, Innsbruck SPIE, P.O. Box 10, Bellingham, Wash. 98227

22-26 Laser Safety: Hazard, Inspection \& Control course, Chicago Laser Inst. of Amer., M. McHenry, 5151 Monroe St., Suite 118W, Toledo, Oh. 43623

May

4-9 5th Int. Symp. on Silicon Materials Science \& Tech., Boston Monsanto Electronic Materials Co., 755 Page Mill Rd., P.O. Box 10123, Palo Alto, Calif. 94303

39th Ann. SPSE Conf., Minneapolis SPSE, $7003 \mathrm{Kil}$ worth La., Springfield, Va. 22151

\section{June}

$2-6$

Optical \& Electro-Optical Engineering Symp., Quebec SPIE, P.O. Box 10, Bellingham, Wash. 98227

9-13 Quantum Electronics Int. Conf., San Francisco OSA Mtgs. Dept., 1816 Jefferson Pl., N.W., Wash., D.C. 20036

9-13 Conf. on Lasers \& Electro-Optics (CLEO '86), San Francisco OSA Mtgs. Dept., 1816 Jefferson Pl., N.W., Wash., D.C. 20036

$16-19$

Ultrafast Phenomena, OSA Top. Mtg., Snowmass, Colo. OSA Mtg. Dept., 1816 Jefferson Pl., N.W., Wash., D.C. 20036

July

$7-11$

Optical Computing Int. Conf., Jerusalem ICO, P.O. Box 4413, 61044 Tel-Aviv, Israel

16-18 Image Detection \& Quality Int. Top. Mtg., Paris $P$. Chavel, Institut d'Optique, B.P. 43, 91406 OrsayCedex, France

\section{August}

$17-22$

30th Ann. Int. Symp. on Optical \& Electro-Optical Eng., San Diego SPIE, P.O. Box 10, Bellingham, Wash. 98227

14-19 Optical \& Electro-Optical Engineering Symp., Cambridge SPIE, P.O. Box 10, Bellingham, Wash. 98227

continued on page 2020 